J Vet Diagn Invest 14:438-441 (2002)

\title{
Neutralizing antibodies against vesicular stomatitis viruses (serotypes New Jersey and Indiana) in horses in Costa Rica
}

\author{
Maren Blickwede, Gaby Dolz, Marco V. Herrero, Sarah M. Tomlinson, Mo Salman
}

\begin{abstract}
Serum samples were collected from domestic horses in 4 different regions of Costa Rica to detect antibodies against vesicular stomatitis viruses, serotypes New Jersey (VSV-NJ) and Indiana (VSV-IN). A total of 214 samples were tested by the virus neutralization test. The sampling regions were identified as low North Pacific dry area (1), low Middle Atlantic humid area (2), low South Pacific humid area (3), and the highlands (4). In region $1,97.1 \%$ of horses were positive for VSV-NJ and $16.5 \%$ were positive for VSV-IN. The mean antibody titer and its standard deviation after logarithmic transformation were $5.86 \pm 0.9$ for VSV-NJ and 3.55 \pm 1.66 for VSV-IN for region 1. In region 2, 40.7\% of horses were positive for VSV-NJ and 32.2\% were positive for VSV-IN. The mean antibody titer in region 2 was $4.33 \pm 1.82$ for VSV-NJ and $3.47 \pm 1.73$ for VSV-IN. In region 3, $20.79 \%$ of horses were positive for VSV-NJ and 27.6\% were positive for VSV-IN. The mean antibody titer in region 3 was $4.39 \pm 1.89$ for VSV-NJ and $3.47 \pm 1.82$ for VSV-IN. In region $4,91.3 \%$ of horses were positive for VSV-NJ and $73.9 \%$ were positive for VSV-IN. The mean antibody titer in region 4 was $5.77 \pm 1.10$ for VSV-NJ and $4.85 \pm 1.63$ for VSV-IN. This is the first published report of the detection of virus-neutralizing antibodies against VSV-NJ and VSV-IN in horses in Costa Rica.
\end{abstract}

Vesicular stomatitis viruses (VSVs) infect domestic animals including horses, cattle, and swine. Two VSV serotypes are known, New Jersey (VSV-NJ) and Indiana (VSV-IN). ${ }^{14}$ The Indiana serotype consists of 3 strains: IN1, IN2 (Cocal), and IN3 (Alagoas).

The recent epidemics of VS in North America were caused by the VSV-NJ serotype in 1995 and 1997 and the VSV-IN serotype in 1997 and 1998. New Mexico, Utah, Arizona, Wyoming, Texas, and Colorado all reported VSVseropositive animals during some outbreak years. An animal was considered positive for VSV in these outbreaks if it had clinical signs consistent with VSV and positive virus isolation, or was positive to 1 or more serologic tests, or both. Overall, $99 \%$ of the confirmed cases occurred in horses with Colorado reporting the largest number of equine VS cases. ${ }^{6}$ The International Office for Epizootics classifies VS as a List A disease. This classification requires reporting of VS cases to the international agriculture community and results in the restriction of animal movements from within affected areas. ${ }^{15}$ Vesicular stomatitis outbreaks may have significant impacts on horse and livestock industries.

Vesicular stomatitis is a disease of the western hemisphere and is endemic on Ossabaw Island, Georgia, and in Mexico, Central America, and South America.4,10,12 Vesicular stomatitis is very important to cattle industries because it is clinically indistinguishable from foot and mouth disease (FMD). Foot and mouth disease has been eradicated from most coun-

From Universitaet Hannover, Welfengarten 1, 30169 Hannover, Germany (Blickwede), PCVET, Escuela de Medicina Veterinaria, Universidad Nacional Apdo 304-3000 Heredia, Costa Rica (Dolz, Herrero), and the Center of Veterinary Epidemiology and Animal Disease Surveillance Systems, College of Veterinary Medicine and Biomedical Sciences, Colorado State University, Fort Collins, CO 80523-1676 (Tomlinson, Salman).

Received for publication July 6, 2001. tries in the western hemisphere; therefore, serologic distinction between the 2 diseases is critical.

In horses, VS manifests as vesicles and ulcerations on the tongue, mucosal surfaces in the mouth, and the coronary bands. ${ }^{3}$ Equids are susceptible to both VSV-NJ and VSV-IN, but are not affected by FMD. No reports have been published concerning VS in horses in Costa Rica.

From 1991 to 1999, information on only 10 cases of equine VS has been recorded in the archives of the Laboratory of Virology, Tropical Diseases Research Program, School of Veterinary Medicine, Universidad Nacional, Heredia, Costa Rica. A case was defined as clinical disease with virus isolation. The occurrence of these cases was reported in the following provinces: 3 in Heredia in October 1994, in Alajuela in March 1994, 4 in Cartago in February 1996, 1 in Alajuela in February 1996, and 1 in Heredia in February 1996. The objective of this study was to determine the level of antibodies against VSV-NJ and VSV-IN in horses from different regions of Costa Rica.

Blood samples were collected from 214 mixed-breed horses on 19 farms in Costa Rica. All available horses were sampled on each farm visited. Farms were located in 4 regions: low North Pacific dry area (region 1), low Middle Atlantic humid area (region 2), low South Pacific humid area (region 3), and the highlands (region 4). Regions of Costa Rica are defined in Figure 1. Details of the farms and horses sampled are provided in Table 1.

Serum samples were collected between October 1998 and May 1999. Blood was drawn from the jugular vein of each horse with a needle and vacutainer tube. Samples were transported to the laboratory and centrifuged for 10 minutes at $5,000 \times g$, and the serum was stored at $-20 \mathrm{C}$ until tested. The serum was analyzed by the virus-neutralization test (VNT) for both VSV-NJ and VSV-IN.

The VNT was performed as previously described. ${ }^{10} \mathrm{~A}$ microtiter neutralization test was used for the detection of neu- 


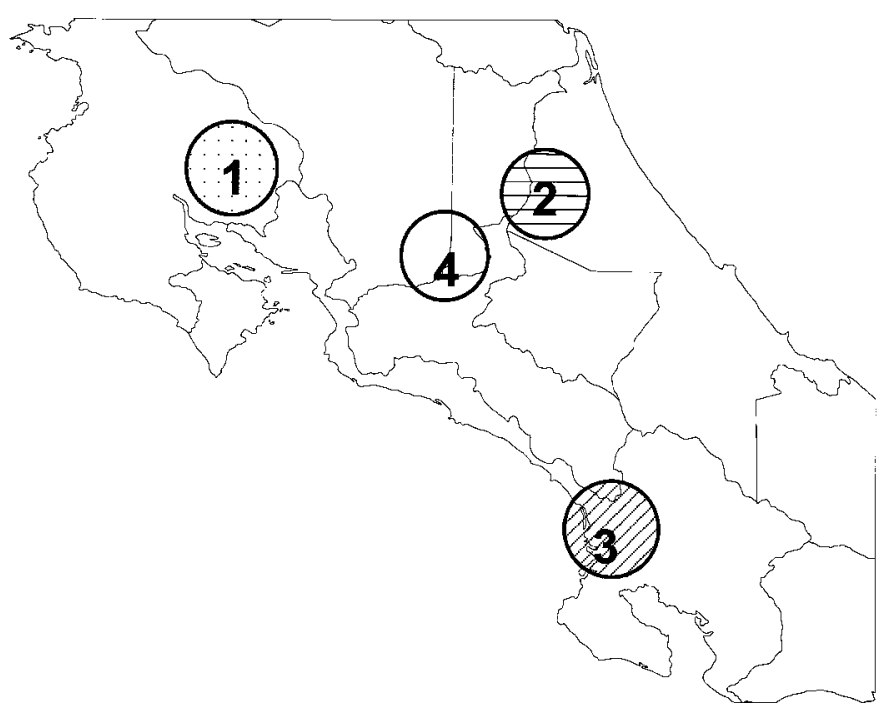

100000

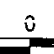

100000

200000

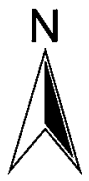

Figure 1. Costa Rican regions for sampling horses for evidence of vesicular stomatitis virus (note: 1, 2, 3, and 4 refer to study regions; see Table 1).

tralizing antibodies to VSV-NJ and VSV-IN. Eight serum samples were heat inactivated ( $56 \mathrm{C}$ for 30 minutes), diluted from 1:100 to 1:6,400 (4-fold dilutions) with Dulbecco minimum essential medium in duplicate wells of tissue culture plates, and 100-200 median tissue culture infective doses of VSV-NJ (Greentree strain) and VSV-IN (Cuhayo strain) were added to each well. After incubation of the plates at $37 \mathrm{C}$ and $5 \% \mathrm{CO}_{2}$ for 1 hour, Vero E6 cells were added as indicator cells $(10,000$ cells/well). Cell control, virus control, positive control, and negative control serum samples were included in each neutralization assay. All plates were read in 48 hours, and each sample for each serotype (New Jersey or Indiana) was determined to be positive if a confluent monolayer of Vero cells appeared at a dilution of 1:8 or greater.

The percentage of seropositive horses was defined as the number of positive samples for each serotype, divided by the number of samples for that region. The log-likelihood chi-square test was used to compare the proportion of seropositive horses between regions (Table 1). Antibody titers were transformed by taking the natural log of the end titer dilution. The mean and the standard deviation for the transformed titer data were calculated. The 4-fold dilutions used for the VNT results in the titer data were considered noncontinuous. Therefore, the Kruskal-Wallis test, which performs an analysis of the ranks of the data, was used to compare the mean titers of VSV-NJ and VSV-IN between regions. ${ }^{11}$ The results of these comparisons are provided in Table 2. All statistical tests were conducted with statistical software. ${ }^{a}$

Previous studies in Costa Rica reported that VSV-NJ infections occur much more frequently than VSV-IN infections in dairy cattle. ${ }^{10}$ Our findings concur, with $70.6 \%$ of horses serologically positive for VSV-NJ and $28.5 \%$ positive for

Table 1. Characteristics and percent seropositive for vesicular stromatitis virus, serotype New Jersey (VSV-NJ) and serotype Indiana (VSV-IN) for horses sampled in 4 regions of Costa Rica.

\begin{tabular}{|c|c|c|c|c|c|c|c|c|}
\hline Region & Total farms & Total horses & No. males & No. females & $\begin{array}{l}\text { No. horses } \\
\leq 5 \text { years }\end{array}$ & $\begin{array}{c}\text { No. horses } \\
>5 \text { years }\end{array}$ & $\begin{array}{l}\% \text { positive } \\
\text { VSV-NJ }\end{array}$ & $\begin{array}{l}\% \text { positive } \\
\text { VSV-IN }\end{array}$ \\
\hline 1 & 3 & 103 & 32 & 71 & 23 & 81 & 97.1 & 16.5 \\
\hline 2 & 2 & 59 & 30 & 29 & 14 & 45 & 40.7 & 32.2 \\
\hline 3 & 7 & 29 & 20 & 9 & 3 & 26 & 20.7 & 27.6 \\
\hline 4 & 7 & 23 & 13 & 10 & 0 & 23 & 91.3 & 73.9 \\
\hline Totals & 19 & 214 & 95 & 119 & 40 & 175 & $70.6^{*}$ & $28.5 \dagger$ \\
\hline
\end{tabular}

$* \chi^{2}=99.7, P<0.0001$.

$\dagger \chi^{2}=30.9, P<0.0001$. 
Table 2. Means of transformed virus-neutralization antibody titers for vesicular stromatitis virus, serotype New Jersey (VSV-NJ) and serotype Indiana (VSV-IN) in horses in 4 regions of Costa Rica.

\begin{tabular}{ccc}
\hline \hline Region & VSV-NJ mean titer $(\mathrm{SD}) *$ & VSV-IN mean titer $(\mathrm{SD}) \dagger$ \\
\hline 1 & $5.86(0.90)$ & $3.55(1.66)$ \\
2 & $4.33(1.82)$ & $3.47(1.73)$ \\
3 & $4.39(1.89)$ & $3.47(1.81)$ \\
4 & $5.77(1.10)$ & $4.85(1.63)$ \\
\hline
\end{tabular}

* Kruskal-Wallis test for VSV-NJ: $\chi^{2}=25.5, P<0.0001$.

$\dagger$ Kruskal-Wallis test for VSV-IN: $\chi^{2}=7.09, P<0.07$.

VSV-IN. Previous research also proposed the existence of 2 foci of VS, 1 located at a dry, low elevation (region 1) and the other at a higher elevation (region 4$).{ }^{9}$ We found that in regions 1 and 4, more than $90 \%$ of horses tested had virusneutralizing antibodies to VSV-NJ. In regions 2 and 3, respectively, 40.7 and $20.7 \%$ of horses had virus-neutralizing antibodies to VSV-NJ. The percentage positive between regions was statistically different for VSV-NJ $\left(\chi^{2}=99.7, P<\right.$ $0.0001)$. The greatest and least percent positive for VSV-IN were found in region $4(73.9 \%)$ and region $1(16.5 \%)$, respectively. In regions 2 and 3, respectively, 32.2 and $27.6 \%$ of horses had virus neutralizing antibodies to VSV-IN. The percentage positive between regions was statistically different for VSV-IN $\left(\chi^{2}=30.9, P<0.0001\right)$. Differences in percent positives among the regions may be associated with the different ecological conditions found in each region. Variations in temperature, total precipitation, timing of precipitation, elevation, and vegetation all effect diversity, density, and activity of insect species. Region 4, which had high numbers of seropositive horses for both VSV-NJ and VSVIN, is premontane wet forest with an average annual rainfall of $>2,000 \mathrm{~mm}$ and temperatures ranging from 15 to $30 \mathrm{C}$. Phlebotamine sand flies, known vectors of VSVs, have been collected from farms in this region. ${ }^{5}$ Although a reservoir of VSVs has yet to be identified, a small mammal is the most likely reservoir of VSV. Regional variations in ecologies would result in differences in populations of small mammals, both in density and species in each region. Research that focuses on the prevalence, population, and requirements of specific vectors in each region is warranted to understand the importance of insects and rodents in the transmission of VS throughout Costa Rica.

This report describes an exploratory study to determine the presence of virus-neutralizing antibodies against VSVNJ and VSV-IN in horses within different regions of Costa Rica. Premises included in this study may not be representative of all premises with horses in the regions selected. The relationship of farm factors (management, location, land use, and other animal species), host factors (age, breed, gender, geographic origin, and travel history), ecologic factors (precipitation, temperature, and humidity), and insect vector and rodent factors (presence and prevalence of speculated VSV transmitters) to the presence of antibodies were not examined in this study. Further examination of these factors may explain the significant difference in frequency of VSVNJ and VSV-IN antibody titers and lead to the identification of those factors that contribute to the variability between regions.

All previous studies of VS in Costa Rica have focused on the disease in cattle. ${ }^{1,2,7,8,10,13}$ Horses, pigs, and humans live in the same areas as cattle and all may be exposed to similar risk factors. More specific studies are required to determine the serologic status of other domestic animals and their role in the transmission of VSV in endemic areas.

Acknowledgements. We appreciate the help provided by Dr. Carlos Jiménez, head of the Virology Laboratory in Costa Rica. Thanks to our field collectors, laboratory technicians, and graduate students for their participation in the study. We appreciate the support of Dr. Frederick Haas, (Virologisches Institut, Tieraerzliche Hoschschule Hannover, Hannover, Germany). We recognize the German Academic Exchange Service (DAAD) and the Tieraerztliche Hochschule Hannover for additional funding. This investigation was supported by grant FG-CR-106 (CS-AES-5) from the United States Department of Agriculture and the Center of Economically Important Infectious Animal Diseases of Colorado State University, Fort Collins, Colorado, through a special grant from USDA: CSREES.

\section{Sources and manufacturers}

a. SAS Version 8, SAS Institute, Cary, NC.

\section{References}

1. Atwill ER, Rodríguez LL, Hird DW, Rojas O: 1993, Environmental and host factors associated with seropositivity to New Jersey and Indiana vesicular stomatitis viruses in Costa Rican cattle. Prev Vet Med 15:303-314.

2. Cornelisen BPM, Pérez E: 1987, Algunos aspectos del comportamiento epidemiológico de la estomatitis vesicular (EV) en Costa Rica. Estudio retrospectivo: 1972/1986. Ciencias Vet IX(2-3):143-151.

3. Green SL: 1993, Vesicular stomatitis in the horse. Vet Clin North Am Equine Pract 9:349-353.

4. Hernández De Anda J, Salman MD, Mason J, et al.: 1992, A prospective study of vesicular stomatitis in cattle in an enzootic region of México. Prev Vet Med 14:209-215.

5. Herrero MC, Jimenez AE, Rodriguez LL, Pereira R: 1994, Phlebotomines (Diptera: Psychodidae) collected at a Costa Rican dairy farm in a vesicular stomatitis endemic area. J Med Entomol 31:912-914.

6. McCluskey B, Mumford E, Traub-Dargatz J, Salman, MD: 1999, Vesicular stomatitis outbreaks in the southwestern United States: 1995-1998. Proc Soc Trop Vet Med 1999:97.

7. Pérez E, Cornelissen B: 1988, Retrospective study of the temporal distribution of vesicular stomatitis in cattle in Costa Rica, 1972-1986. Prev Vet Med 6:1-8.

8. Remmers L, Pérez E, Jiménez A, et al.: 2000, Longitudinal studies in the epidemiology of vesicular stomatitis (VS) on Costa Rican dairy farms. Ann N Y Acad Sci 916:417-430.

9. Rodriguez LL, Fitch WM, Nichol ST: 1996, Ecological factors rather than temporal factors dominate the evolution of vesicular stomatitis virus. Proc Natl Acad Sci USA 93:13030-13035.

10. Rodriguez LL, Vernon S, Morales AI, Letchworth GJ: 1990, Serological monitoring of vesicular stomatitis New Jersey in enzootic regions of Costa Rica. Am J Trop Med Hyg 42:272281.

11. Shott S: 1990, Statistics for health professionals. WB Saunders, Philadelphia, PA. 
12. Stallnecht, DE: 1999, VSV-NJ on Ossabaw Island, Georgia: the truth is out there. Proc Soc Trop Vet Med 1999:131.

13. Vanleeuwen JA, Rodríguez LL, Waltner-Toews D: 1995, Cow, farm and ecologic risk factors of clinical vesicular stomatitis on Costa Rican dairy farms. Am J Trop Med Hyg 53:342-350.
14. Webb PA, Holbrook FR: 1989, Vesicular stomatitis. In: The arboviruses: epidemiology and ecology, ed. Monath TP, vol. 5, pp. 2-23. CRC Press, Boca Raton, FL.

15. Office International des Epizooties. 2001, World Animal Health in 2000. Paris, France.

J Vet Diagn Invest 14:441-444 (2002)

\title{
Enteric coronavirus infection in a juvenile dromedary (Camelus dromedarius)
}

\author{
Arno Wünschmann, Rodney Frank, Kem Pomeroy, Sanjay Kapil
}

\begin{abstract}
A case of an enteric coronavirus infection in a 6-week-old dromedary calf is described. The animal had diarrhea for 5 days and died despite symptomatic treatment. Numerous viral particles, approximately $140 \mathrm{~nm}$ in diameter, with club-like projections were detected in the feces by electron microscopy. These characteristics were consistent with a coronavirus. Immunohistochemical reactivity with 2 antigenic group II coronavirus-specific antibodies confirmed the presence of viral antigen in colonic epithelial cells. The death of the animal was attributed to a neutrophilic and emphysematous colitis that likely was caused by an infection with a Clostridium sp.
\end{abstract}

A 6-week-old female dromedary (Camelus dromedarius) calf with a history of acute diarrhea died 5 days after the initial symptoms. According to the owner, the calf was born in Missouri on a pasture that was also inhabited by zebras. The camel nursed from its mother for 1 week after birth. At 1 week of age, the calf was separated from its mother and shipped to a farm in Wisconsin. Upon arrival, the calf was contained indoors and initially fed approximately 1 liter of warm calf milk replacer ${ }^{\mathrm{a}}$ containing oxytetracycline and neomycin 4 times per day. The amount of milk replacer was gradually increased to approximately 2 liters per feeding. Clover grass hay was available at all times. The calf had contact with miniature horses, zebras, and reindeers that were kept in the same barn in different stalls. The calf was healthy for approximately 4 weeks. Its weight was approximately $75 \mathrm{~kg}$. Five days before death, the calf developed watery diarrhea after initially being bloated. The owner administered penicillin (approximately 20,000 units $/ \mathrm{kg}$ ) intramuscularly. The calf was presented to the referring veterinarian for the first time approximately 1 day after the initial signs were noted by the owner. The animal appeared to be mildly dehydrated. The calf was treated by the veterinarian with butorphanol, ${ }^{\mathrm{b}}$ dipyrone, ${ }^{\mathrm{c}}$ and flunixin meglumine ${ }^{\mathrm{d}}$ intravenously and received electrolytes per os because it was still drinking. At the second visit 2 days later, the rectal temperature was approximately $35 \mathrm{C}$ (reference range $^{7}$ : 36-40 C). The hematocrit was slightly elevated (37.5\%; reference range $\left.^{13}: 26-31 \%\right)$. The calf was tachycardic (60 heart beats/

From the Department of Veterinary Diagnostic Medicine, College of Veterinary Medicine, University of Minnesota, 1333 Gortner Avenue, St. Paul, MN 55108 (Wünschmann, Frank, Pomeroy), and the Department of Diagnostic Medicine-Pathobiology, College of Veterinary Medicine, 1800 Denison Avenue, Manhattan, KS 66506 (Kapil).

Received for publication October 1, 2001. minute; reference range $^{7}: 40-50$ beats/minute). The white blood cell count was 16,000 leukocytes $/ \mathrm{ml}$ (reference range $\left.^{13}: 13,000-24,000\right)$ with neutrophilia (83\%; reference range $^{13}$ : $\left.53-74 \%\right)$. The anti-inflammatory and antibiotic treatment was continued but the calf died and was submitted to the Department of Veterinary Diagnostic Medicine, University of Minnesota, St. Paul, Minnesota, for postmortem examination. Tissue samples, including small and large intestine, lung, brain, liver, kidney, spleen, heart, intestinal lymph node, and adrenal gland, were fixed in $10 \%$ buffered formalin and embedded in paraffin. Sections cut at $4 \mu \mathrm{m}$ were stained with hematoxylin and eosin (HE). Additional sections of the intestine were stained with a modified Gram stain. Fresh samples of lung and liver were submitted for aerobic culture. The intestine was cultured under aerobic and anaerobic conditions by routine laboratory procedures. Feces were submitted for routine parasitologic examination by flotation techniques. Fecal samples were examined for viruses by using direct negative-contrast transmission electron microscopy as previously described. ${ }^{8}$

Avidin-biotin-peroxidase complex method was used for immunohistochemical demonstration of coronaviral and rotaviral antigens as previously described., ${ }^{4,19}$ Two monoclonal antibodies against ruminant coronavirus (bovine and elk coronavirus) spike protein ${ }^{\mathrm{e}}$ (clone Z3A5) and nucleocapsid protein $^{\mathrm{f}}$ (clone $8 \mathrm{~F} 2$ ), 1 monoclonal antibody against porcine transmissible gastroenteritis virus ${ }^{\mathrm{g}}$ (TGE; clone 14-E3), and 1 monoclonal antibody against bovine rotavirus ${ }^{\mathrm{h}}$ (clone 910) served as primary antibodies. ${ }^{3,16,19}$

At necropsy, the calf was in a good nutritional state. The colon and cecum were moderately distended and filled with yellow-brown watery fluid. The mucosa of the proximal colon and cecum had multiple, slightly raised, well-demarcated, red-white mottled areas, which were up to $10 \mathrm{~cm}$ in length by $5 \mathrm{~cm}$ in width (Fig. 1). The mesentery was edem- 